\title{
Síndrome Neuroléptica Maligna: um relato de caso
}

\author{
Débora Bernardes Peixoto ${ }^{1}$ \\ Joaquim Dias da Costa Neto ${ }^{2}$ \\ Flávia Campos ${ }^{3}$ \\ Jordana Rey Laureto ${ }^{4}$ \\ Talles Borges Pereira ${ }^{5}$
}

\footnotetext{
${ }^{1}$ Médica residente de clínica médica do Hospital Regional de Sobradinho, Distrito Federal, Brasil;

${ }^{2}$ Médico especialista em clínica médica pela Universidade Federal de Uberlândia, Minas Gerais, Brasil

${ }^{3}$ Médica especialista em clínica médica, preceptora da residência de Clínica Médica do Hospital Regional de Sobradinho, Distrito Federal, Brasil.

${ }^{4}$ Médica especialista em intensivismo, preceptora da residência de Clínica Médica do Instituto Hospital de Base, Distrito Federal, Brasil

${ }^{5}$ Médico especialista em gastroenterologia, Distrito Federal, Brasil.
}

Endereço eletrônico para correspondência: debora.bernardesp@hotmail.com

\section{RESUMO}

Introdução: Síndrome neuroléptica maligna é uma doença rara, observada principalmente em homens de meia idade antes da $4^{\text {a }}$ década de vida, caracterizada por flutuação do nível de consciência, rigidez muscular intensa, disâutonomia (variação de pulso, pressão arterial (PA) e frequência respiratória) e hipertermia. O diagnóstico é clinico e laboratorial, devendo ser excluídas outras causas. Descrição: D.G.S., 43anos, masculino, casado. Apresentou quadro de surto psicótico sendo medicado com haloperidol e prometazina, evoluindo posteriormente com quadro de rigidez muscular, sudorese, hipertermia, alteração neurológica e elevação de creatinina fosfoquinase (CPK), compatível com o diagnóstico de Síndrome Neuroléptica Maligna, provavelmente secundário a infusão de haloperidol. Conclusão: A Síndrome Neuroléptica Maligna se caracteriza por uma reação ao uso de neurolépticos e leva o indivíduo a apresentar múltiplas alterações orgânicas, possivelmente fatais. O diagnóstico é clínico e laboratorial. O tratamento primordial é a suspensão da droga causadora, não sendo ainda estabelecido um tratamento específico.

Palavras-chave: Síndrome Neuroléptica Maligna, fármacos neurolépticos, neurologia, psiquiatria

\section{ABSTRACT}

\section{Neuroleptic Malignant Syndrome: a case report}

Introduction: Neuroleptic malignant syndrome is a rare disease, observed mainly in middle-aged men before the 4th decade of life, characterized by fluctuations in the level of consciousness, intense muscle stiffness, dysautonomia (variation in pulse, blood pressure (BP) and respiratory rate) and hyperthermia. The diagnosis is clinical and laboratory, and other causes must be excluded. Description: D.G.S., 43 years old, male, married. He presented with a psychotic outbreak and was medicated with haloperidol and promethazine, subsequently evolving with muscle stiffness, sweating, hyperthermia, neurological changes and elevated creatine phosphokinase (CPK) compatible with the diagnosis of Malignant Neuroleptic Syndrome, probably secondary to haloperidol infusion. Conclusion: The Neuroleptic Malignant Syndrome is characterized by a reaction to the use of neuroleptics and leads the individual to present multiple organ changes, possibly fatal. The diagnosis is clinical and laboratory. The primary treatment is the suspension of the causative drug, and a specific treatment has not yet been established.

Keywords: Neuroleptic Malignant Syndrome, neuroleptic drugs, neurology, psychiatry

\section{INTRODUÇÃO}

A síndrome neuroléptica maligna (SNM) é uma patologia relacionada ao uso de medicamentos com efeitos antagonistas aos receptores D2 de dopamina. Está mais comumente associada ao uso de 
neurolépticos e antipsicóticos de alta potência, como fenotiazina e butirofenonas, entretanto, também tem sido apresentada com agentes atípicos como risperidona, quetiapina e olanzapina, metoclopramida e após a retirada de anti-dopaminérgicos ${ }^{(1)}$. Trata-se de um efeito adverso raro, porém com elevada gravidade. Essa entidade clínica foi reconhecida por volta dos anos $60^{(2,3)}$.

A doença se apresenta com características clássicas, compreendendo flutuação do nível de consciência, rigidez muscular intensa, disâutonomia (variação de pulso, PA e frequência respiratória) e hipertermia. As alterações laboratoriais consistem em leucocitose, elevação de enzimas hepáticas e aumento da creatinina fosfoquinase (CPK). Pode haver elevação de escórias nitrogenadas (devido à insuficiência renal associada), além de que mioglobinúria, aldolase e desidrogenase láctica, ocasionalmente, podem estar elevados ${ }^{(3)}$.

A incidência dessa síndrome encontra-se por volta de $0,02 \%$ e $0,03 \%$. Dentre as possibilidades de diagnóstico diferencial estão a hipertermia maligna, síndrome serotoninérgica, raiva, tétano, delirium, feocromocitoma e tireotoxicose ${ }^{(1)}$.

A SNM é uma complicação idiossincrática, apresentando uma evolução rápida, grave e potencialmente fatal caso não seja adotado o manejo adequado ${ }^{(1)}$.

O manejo desse paciente deve ser realizado em ambiente de terapia intensiva com suspensão imediata do fármaco que causou a reação ${ }^{(4)}$. O tratamento farmacológico ainda permanece controverso, porém existem estudos que mostram benefícios com o uso de benzodiazepínicos, agentes colinérgicos como bromocriptina e amantadina, além do bloqueador neuromuscular dantrolene ${ }^{(5,6)}$.

O prognóstico, a morbidade e a mortalidade são variáveis e dependem de múltiplos fatores, sendo o principal deles o diagnóstico e tratamento precoce. Atualmente pode-se encontrar na literatura uma variação de mortalidade entre 9 a $30 \%{ }^{(6)}$.

O presente relato descreve o caso de um paciente de 43 anos que apresentou SNM associada ao uso de haloperidol e seu recente tratamento intra-hospitalar em setembro de 2020.

\section{DESCRIÇÃO DO CASO}

D.G.S., 43 anos, masculino, casado, reside com a esposa e dois filhos, profissão lombador em frigorífico, natural de Afogados da Ingazeira - PE, e residente de Santa Maria - DF, deu entrada no 
Instituto Hospital de Base (IHBDF) em setembro de 2020, encaminhado do Hospital São Vicente de Paula (HSVP) por apresentar surto psicótico associado a alterações clínicas e laboratoriais.

Paciente iniciou quadro de alteração de comportamento um mês antes da internação com sintomas de insônia, agitação psicomotora, agressividade, alucinações auditivas e discurso místico delirante. Evoluiu com piora significativa, quando tentou enforcar e matar a esposa acreditando que a mesma era seu chefe. Foi levado a Unidade de Pronto Atendimento (UPA) do Novo Gama, por duas vezes, com apoio do Serviço de Atendimento Móvel de Urgência (SAMU). Recebeu haloperidol repetidas vezes e recebeu alta hospitalar. Retornou ao HSVP com manutenção do quadro e foi então transferido ao IHBDF.

Sobre antecedentes pessoais, familiar afirma um primeiro surto psicótico em 2004. Afirma que antes de 2004 ele não apresentava alterações psiquiátricas, mas após esse surto ele permaneceu com alterações como mussitação, insônia e autoagressão, porém não heterodirigida.

Fez seguimento ambulatorial, mas perdeu o seguimento um ano após. Permaneceu relativamente bem até um mês atrás, sem medicação, mantendo as atividades de vida diária, emprego e vigilância dos filhos. Além disso, família nega tabagismo etilismo e uso de substâncias psicoativas, como drogas ilícitas. Negam ainda, qualquer outra comorbidade, como hipertensão arterial sistêmica, diabetes mellitus e dislipidemia.

Inicialmente o paciente foi recebido pela psiquiatria, encontrando-se contido em 4 pontos, olhar fixo, hipoativo, vígil, hipotenaz, em mutismo, desidratado, pele avermelhada, anictérico, acianótico, febril e hipertenso. Com relato de febre nas 12 horas que precediam. Abdome timpânico, com agitação à palpação, aparentemente rigidez de defesa. Exame do estado mental prejudicado. Aos exames laboratoriais apresentava-se com transaminase glutâmico-oxalacética (TGO) 661 e transaminase glutâmico-pirúvica (TGP) 278, alteração de escórias nitrogenadas com creatinina (Cr):1,81 e ureia (Ur): 130. Leucocitose de 18.450 com $2 \%$ de bastonetes. Sódio (Na):160, CPK:25.188, ácido úrico:11,3 e desidrogenase lática (DHL):1864;

Foi então transferido para a Unidade de Clínica Médica e em 2 dias evoluiu com quadro de rigidez muscular, sobretudo em membros superiores, sudorese, hipertermia, alteração neurológica e elevação de CPK compatível com o diagnóstico de Síndrome Neuroléptica Maligna, provavelmente secundário 
a infusão de haloperidol. Ademais, paciente também apresentou quadro de rabdomiólise e pneumonia aspirativa associadas.

Foi instituído tratamento para o quadro de Síndrome Neuroléptica Maligna com dantrolene por 48 horas e midazolam em caso agitação. Para rabdomiólise foi iniciada hidratação venosa vigorosa, reposição de bicarbonato (paciente com $\mathrm{pH}<7,5$ e bicarbonato: 20) e para pneumonia aspirativa foi mantido antibioticoterapia com piperaciclina+tazobactam. Além de correção de distúrbios hidroeletrolíticos.

A despeito das medidas tomadas, no mesmo o dia o paciente evoluiu com desconforto respiratório, diurese protraída e indícios de má troca alvéolo-pulmonar à gasometria (relação $\mathrm{PaO} 2 / \mathrm{FiO} 2: 70$ ), sendo necessária a intubação orotraqueal. Paciente manteve estabilidade hemodinâmica e foi iniciado a sedoanalgesia com midazolam e fentanil.

Foi transferido à UTI onde permaneceu o tratamento com dantrolene por 48 horas. Apresentou alguns períodos de instabilidade hemodinâmica com necessidade de uso de drogas vasoativas em doses baixas, com desmame posterior. Apresentou boa resposta clínica e foi extubado sem intercorrências após 4 dias de internação em Unidade de Terapia Intensiva (UTI). Foi avaliado pela psiquiatria que iniciou uso de diazepam $5 \mathrm{mg}$ 1x/dia e manteve contra indicação absoluta de qualquer tipo de antipsicótico (neuroléptico).

Clinicamente paciente manteve quadro de alteração psiquiátrica, com necessidade de contensão em membros superiores. Pensamento empobrecido, comunicação incompreensível, sensopercepção indeterminada. Respondendo aos questionamentos da entrevista apenas com monossílabos incompreensíveis. Respirando em ar ambiente e hemodinâmica estável sem uso de drogas vasoativas. Apresentando melhora laboratorial com TGO:94, TGP:86, Cr:0,68, Ur:34, Na:146, Leucócitos: 11.270 sem desvio à esquerda e CPK: 8.723.

Deste modo, após a resolução do quadro de síndrome neuroléptica maligna, paciente recebeu alta da unidade de terapia intensiva e foi encaminhado aos cuidados da psiquiatria para finalizar a terapêutica específica para seu distúrbio. 


\section{DISCUSSÃO}

A Síndrome Neuroléptica Maligna é uma reação ao uso ou retirada de medicamentos com efeitos antagonistas aos receptores D2 de dopamina. O diagnóstico é realizado a partir de critérios clínicos e laboratoriais. Ressalta-se que devem ser excluídas outras possíveis causas para sintomas semelhantes, sendo o diagnóstico diferencial o grande desafio dessa patologia.

De acordo com a Associação Americana de Psiquiatria, no Diagnostic and Statistical Manual of Mental Disorders (DSM-IV.) ${ }^{(7)}$, são necessários rigidez muscular severa e febre acompanhado de no mínimo 2 dos 10 itens seguintes: diaforese, disfagia, tremor, incontinência, alteração do estado mental, mutismo, taquicardia, pressão arterial elevada ou lábil, leucocitose, creatinofosfoquinase elevada ${ }^{(7,8)}$. Pode-se notar que o paciente do caso descrito apresentou todos os critérios necessários para confirmar o diagnóstico.

A incidência da síndrome neuroléptica maligna tem variado no decorrer dos anos, principalmente devido a descoberta de novas medicações neurolépticas. Essa taxa reduziu de 0,2\% a 3,2\% nos estudos de 1966 e 1997 para 0,01 a $0,02 \%$ dos pacientes psiquiátricos ${ }^{(2)}$.

Dentre os agentes farmacológicos causadores da síndrome, o mais frequente descrito é o haloperidol. Lavie e col. mostraram em seu estudo o uso de haloperidol em 55\% dos 55 casos de SNM relatados ${ }^{(11)}$. No presente relato o agente responsável por deflagrar a crise foi justamente o haloperidol. Além do haloperidol, atualmente encontram-se estudos com relatos de SNM por uma gama variada de fármacos como flufenazina, lítio, tioridazina, clozapina, metoclopramida, tetrabenazine, ecstasy, carbamazepina, entre outras ${ }^{(9)}$. Existe ainda um relato na literatura de síndrome desencadeada pelo uso de olanzapina.

Existem vários fatores de risco que predispõem ao aparecimento de crises, dentre eles estão: episódio anterior de SNM; início de tratamento; rápido aumento de doses; associação com lítio; presença de síndrome mental orgânica (demência, alcoolismo) indivíduos desnutridos e desidratados; exaustão física (mania, estresse físico intenso, estresse por temperatura); esquemas de polifarmácia; sexo: relação de 2:1 entre homens e mulher; faixa etária: $80 \%$ tem menos de 40 anos, idade média 26 anos. Além desses, ainda existem os fatores de risco relacionados aos fármacos, que são neurolépticos de alta potência (mesmo em doses baixas); neurolépticos de ação prolongada (tais 
medicamentos devem ser evitados no início de tratamento). Dentre os distúrbios psiquiátricos de maior ocorrência estão a esquizofrenia $50 \%$ e distúrbios afetivos $25 \%{ }^{(11)}$. No quadro apresentado podemos elencar como fatores de risco presentes o sexo masculino e o uso de neuroléptico de alta potência.

A fisiopatologia da SNM ainda não foi totalmente compreendida, porém sabe-se que a existe relação intrínseca ao metabolismo da dopamina. A crise é deflagrada exatamente e principalmente por fármacos que tem ação antagonista ao receptor D2 da dopamina. Ademais, os agonistas da dopamina têm apresentado resposta no tratamento da SNM e contribuem para a remissão quando retirados. Existem teorias que apontam para a ocorrência de SNM-like durante a interrupção dos tratos dopaminérgicos das regiões afetadas, como os corpos mamilares, o giro do cíngulo e o núcleo paraventricular hipotalâmico ${ }^{(10)}$.

As manifestações clínicas mais comuns são rigidez muscular, alteração do nível de consciência, disâutonomia e hipertermia. Já a alteração laboratorial mais relevante é o aumento da CPK normalmente acima de $1.000 \mathrm{mg} / \mathrm{L}^{(4)}$. Todas as alterações acima foram experenciadas pelo paciente deste quadro relatado.

Por se tratar de uma síndrome com apresentação clínica ampla existem múltiplos diagnósticos diferenciais. Dentre as possibilidades diagnósticas encontra-se a pneumonia seguida de reação distônica generalizada, sendo esse o principal diagnóstico diferencial. Outra possibilidade seria quadro infeccioso que cursa com exacerbação de sinais extrapiramidais pré-existentes e diminuição de nível de consciência. Também devem ser excluídas síndromes de abstinência como delirium tremens e interações medicamentosas. Outra síndrome rara que se apresenta de forma semelhante a SNM é a catatonia letal ${ }^{(10)}$. No caso descrito acima, os referidos diagnósticos diferenciais foram excluídos através de história, exame físico e exames complementares.

Além de toda gravidade inerente à síndrome em si, tal patologia pode apresentar complicações nas mais diversas regiões orgânicas, tais como desidratação, infecção, rabdomiólise, falência renal, insuficiência pulmonar, pneumonia aspirativa, embolia pulmonar e síndrome cerebelar crônica ${ }^{(11)}$. No relato de caso apresentado o paciente cursou com rabdomiólise e falência renal.

A medida primordial de condução e tratamento da SNM é a suspensão imediata da medicação 
neuroléptica que causou a crise. Além disso, devido a comprovada associação com o metabolismo da dopamina é indicada a substituição de anticolinérgicos por agonistas dopaminérgicos. Medicações dopaminérgicas de uso habitual devem ser mantidas, já que sua suspensão pode representar agravo. A piora com a associação do lítio é amplamente descrita e o mesmo medicamento deve ser descontinuado. O tratamento específico ainda permanece controverso, porém podem ser citados o uso de dantrolene e/ou bromocriptina, lisuride, eletroconvulsoterapia, plasmaferese, levodopa intravenosa, carbidopa/levodopa. No relato por nós descrito, foi realizada suspensão imediata do haloperidol e indicado dantrolene por 48 horas, com boa resposta a terapia instituída ${ }^{(12)}$.

Em conclusão ao estudo, pode-se inferir que a SNM é uma síndrome clínica grave e potencialmente fatal, sendo primordial que todas as especialidades médicas tenham conhecimento para adequado reconhecimento, diagnóstico e manejo precoce. Além disso, apesar de rara, existem relatos de ocorrências com drogas atípicas, apontando para necessidade de aumento de vigilância em pacientes que utilizam essas classes de medicações. Trata-se de uma síndrome reversível e o manejo precoce contribui decisivamente para o sucesso terapêutico e restabelecimento funcional dos pacientes acometidos.

Dessa forma, são necessárias pesquisas com objetivo de obter outros métodos para o diagnóstico precoce, tratamento específico da síndrome neuroléptica maligna.

\section{REFERÊNCIAS}

1. Souza RAP, Silva MAF, Coelho DM, Galvão MLS, Souza NAC, Picão AP. Síndrome neuroléptica maligna. Rev Bras Clin Med. 2012; 10(5):40-5.

2. Berman BD. Neuroleptic malignant syndrome: a review for neurohospitalists. Neurohospitalist. 2011 Jan;1(1):41-7. doi: 10.1177/1941875210386491. PMID: 23983836; PMCID: PMC3726098. Disponível em: www.ncbi.nlm.nih.gov/pmc/articles/PMC3726098

3. Kaplan H, Sadock B. Síndrome Neuroléptica Maligna. 6th ed. Tratado de psiquiatria: Artmed; 1999. 2 vol. 
4. Frucht SJ. Treatment of movement disorder emergencies. Neurotherapeutics. 2014 Jan;11(1):20812. doi: 10.1007/s13311-013-0240-3. PMID: 24356784; PMCID: PMC3899491. Disponível em: www.ncbi.nlm.nih.gov/pmc/articles/PMC3899491/

5. Rehman T, deBoisblanc BP. Persistent fever in the ICU. Chest. 2014 Jan;145(1):158-165. doi: 10.1378/chest.12-2843. PMID: 24394828. Disponível em: journal.chestnet.org/article/S00123692(15)49344-4/fulltext

6. Pope HG Jr, Aizley HG, Keck PE Jr, McElroy SL. Neuroleptic malignant syndrome: long-term follow-up of 20 cases. J Clin Psychiatry. 1991 May;52(5):208-12. PMID: 1674508.

7. Gurrera RJ, Chang SS, Romero JA. A comparison of diagnostic criteria for neuroleptic malignant syndrome. J Clin Psychiatry. 1992 Feb;53(2):56-62. PMID: 1347292.

8. Levenson JL. Neuroleptic malignant syndrome. Am J Psychiatry. 1985 Oct;142(10):1137-45. doi: 10.1176/ajp.142.10.1137. PMID: 2863986.

9. Lavie CJ, Ventura HO, Walker G. Neuroleptic malignant syndrome: three episodes with different drugs. South Med J. 1986 Dec;79(12):1571-3. PMID: 2878496.

10. Souza RA, Silva MA, Coelho DM. Síndrome Neuroléptica Maligna. Revista Brasileira de Clínica Médica. 2012;10(5):440-445.

11. Moscovich M, Nóvak F, Fernandes A. Neuroleptic Malignant Syndrome. Archive of Neuropsychiatry. 2011;69(5):751-755.

12. Flaherty J, Chanon R, Davis J. Psiquiatria, diagnóstico e tratamento. Psiquiatria, diagnóstico e tratamento: [publisher unknown]; 1990. 